\title{
Projective Relativity: Present Status and Outlook*
}

\author{
Bertfried Fauser \\ Universität Konstanz \\ Fachbereich Physik, Fach M678 \\ 78457 Konstanz, Germany \\ E-mail: Bertfried.Fauser@uni-konstanz.de
}

October 29, 2000

\begin{abstract}
We give a critical analysis of projective relativity theory. Examining Kaluza's own intention and the following development by Klein, Jordan, Pauli, Thiry, Ludwig and others, we conclude that projective relativity was abused in its own terms. Much more in the case of newer higher dimensional Kaluza-Klein theories with non-Abelian gauge groups. Reviewing the projective formulation of the Jordan isomorphy theorem yields some hints how one can proceed in a different direction. We can interpret the condition $\stackrel{5}{R}{ }_{\mu \nu}=0$ not as a field equation in a 5-dimensional Riemannian space, e.g. as vacuum Einstein-Hilbert equation, but can (or should) interpret it as a geometrical object, a null-quadric. Projective aspects of quantum (field) theory are discussed under this viewpoint.
\end{abstract}

MSC 2000: 83E15

Keywords: Projective relativity, Kaluza-Klein theory, linear complex, null-invariance, Cayley-Klein measure

\section{Introduction}

\subsection{Our Motivation}

The aim of the present note is to oppose projective geometry to such different branches of physics as projective relativity and quantum field theory. We unmask a hidden Riemannian core of projective relativity, the origin of the vacuum field equations, in a polemic way using Hestenes' idea of 'mathematical viruses'. We belief, that only a radical projective point is able to reformulate relativity theory in such a way, that compatibility to quantum field theory can be obtained. In order to to this, we propose the null-quadric to be taken a 'principle' and hence as origin of the 5-dimensional field equations. This argument is valid in 5 dimensional Kaluza-Klein theories only. Projective differential geometry was surprizingly already developed very early [62, 32] even if used marginally later.

Trying to compactify and understand the algebraic structure of quantum field theory, we have been led in a series of papers $16,18,21,15,17,20,23$ to two main results: Graßmann Hopf and Clifford (Hopf?) algebras are the natural language to code quantum field theories. Some singularities simply drop out after a rigorous algebraization;

*This paper is dedicated to Prof. Dehnen on the occasion of his 65 th anniversary. 
and, Clifford geometry and Graßmann-Cayley projective structure are not only related to but part of the core features of quantum field theory.

We try to puzzle together projective relativity and projective quantum field theory to contrast recent belief in quantization of gravity e.g. by super string, brane or Mtheory. However, our arguments are not yet fully developed and a deeper understanding of the Hopf algebraic structure involved in both cases is needed, but first outcomes are summarized in the conclusion.

\subsection{Kaluza's Motivation}

In 1921 a paper by Th. Kaluza on the unity problem of physics 40 found its way into print only after its publication had been postponed several times by Einstein who seemed to have disliked this idea. Kaluza, making a recourse to Weyl, tries to interpret the electromagnetic four potential $A_{\mu}$ and the therefrom derived field strength $F_{\mu \nu}$ as a 'verstümmelte Dreizeigergröße' [truncated Christoffel symbol]. Comparing $1 / 2 F_{\mu \nu}=$ $1 / 2\left(A_{\mu, \nu}-A_{\nu, \mu}\right)$ with $\left[\begin{array}{c}i \lambda \\ \kappa\end{array}\right]=1 / 2\left(g_{i \kappa, \lambda}+g_{\kappa \lambda, i}-g_{i \lambda, \kappa}\right)$ he concluded that one can unite gravity and electromagnetism by the introduction of a fifth world dimension. This leads to the new 5-dimensional 'metric'

$$
\left(\stackrel{5}{g}_{i j}\right):=\left(\begin{array}{cc}
g_{\mu \nu} & A_{\kappa} \\
A_{\lambda}^{T} & \phi
\end{array}\right) .
$$

Kaluza immediately runs into the problem, that physically observed quantities do not depend on the fifth world coordinate. He circumvents this problem by introducing the 'cylinder condition' i.e. the requirement that $\partial_{5}(\ldots)=0$ for any physical quantity.

A second problem is the scalar $\stackrel{5}{g}_{55}=\phi$ which had not an immediate interpretation and was set to one. However, after this artifical steps, Kaluza was able to incorporate the electromagnetism in his 5-dimensional formalism in a beautiful and satisfying way by splitting all equations into 1-4 dimensions and the 5 th one. However, for our point the last sentence in Kaluza's paper is of great importance: "Sollte es sich aber einmal bestätigen, daß mehr hinter den vermuteten Zusammenhängen steckt als nur ein leerer Formalismus, so würde dies entschieden einen neuen Triumph für Einsteins allgemeine Relativitätstheorie bedeuten, um deren sinngemäße Anwendung auf eine fünfdimensionale Welt es sich hier handelt" 40]. [Should more than a presumed formalism be found to reside behind these presumed connections, we would then face a new triumph of Einstein's general relativity, whose appropriate application to a fivedimensional world is our main concern here. [41]] Beside the probable wish to please Einstein, this sentence shows, that Kaluza was not led to his idea by projective reasoning, but by applying once more metrical structures in the additional 5 th dimension. This became much more true in modern non-Abelian extensions of Kaluza-Klein theories.

\subsection{Projective Interpretation}

The beauty and compactness of Kaluza's unification attracted many other scientists to work in this area. Among others, Klein, Veblen, Einstein(!), Jordan, Pauli, Ludwig, Thiry, Schmutzer, Lessner, [38, 50, 51, 45, 55, 43] etc. and of course Dehnen [46] developed a deeper understanding of Kaluza-Klein theories some of them by introducing projective techniques.

In a metrical model of a projective $n$-space one can describe projective 'points' by equivalence classes of $n+1$ tuples of coordinates up to a scalar factor. A projective 4 -space has thus 5 coordinates. An analogous situation occurs in the reduction from 
4-dimensional Minkowski space to 3-dimensional Euclidean space of Newtonian physics. An obvious benefit of such an interpretation is, that one has not to bother about any metrical interpretation of the 5-dimensional space in terms of 'world dimensions'.

The main problem is to relate the projective space to a metrical world continuum in an appropriate way. This was done by Jordan, proving the Jordan isomorphy theorem, which provides a reduction from projective space to the usual Riemannian 4-space. It was also Jordan, who noticed that the new scalar $\phi$ introduced in the 5 -metric can be related to a field and the gravitational 'constant'. This provides a possibility to vary the strength of gravitation. Such a reasoning opened the way for developments as inflation and dynamical cosmological models.

However, the germ of a metrical theory could not be abandoned even in the projective approaches developed so far, since there is a need to come up with field equations in the 5-dimensional space and these field equations are usually modelled in a way close to the Einstein-Hilbert theory i.e. based on a metrical continuum. This will be discussed below in more detail. We can conclude, that the projective interpretation was not fully adopted in Kaluza-Klein theories, which have been seen and treated as geometrical theories all the times.

\section{Present Status -}

\subsection{Mathematical and Physical Viruses}

D. Hestenes introduced in [34] the notion of a mathematical virus (MV), which is an analogy of a biological virus (BV) or a computer virus (CV). "A virus cannot exist by itself, but when attached to a host it replicates repeatedly until it impairs the function of the host, sometimes to a point of disabling the host altogether" 34 p.3. From this definition, it is clear that also ideas (or better ideologies) can be treated as viruses. Hestenes proceeds to describe as species of mathematical viruses the coordinate virus MV/C: 'Coordinates are essential to calculations', the quadratic form virus MV/Q: 'Clifford algebra is the algebra of a quadratic form' which will become important below. Different other species of MV as MV/G, MV/DS, MV/K and MV/T are also described there, and the reader is called to 'infect' himself by reading the article.

We add to this list, as Varags has done recently [59 in an analogous context, two more mathematical or might be physical viruses (PV). The first is the point space virus: PV/P: 'Lines, planes, space, etc. are made from continua of points'.

The antidote to this kind of virus is projective geometry, where one can in principle start from say planes to construct lines and points showing that points can be seen as a continuum of planes. The principle of duality (in projective 3 space) is simply the statement that if one interchanges 'point' with 'plane' and the operations 'join' with 'meet' and vice versa, every theorem for 'points' will hold true for 'planes' and vice versa. The theorem of Brianchon, a dual version of Pascal's theorem is a prominent case, where it took 150 years to find a truth which is a trivial outcome of this principle [39].

Now, Riemannian geometry is intrinsically tied to the primacy of points, since the metric is given infinitesimally at a point on a Riemannian manifold e.g.

$$
\mathrm{d} s^{2}={ }_{g}^{5}{ }_{i j} \mathrm{~d} x^{i} \mathrm{~d} x^{j}=g_{\mu \nu} \mathrm{d} x^{\mu} \mathrm{d} x^{\nu}+g_{5 \mu} \mathrm{d} x^{5} \mathrm{~d} x^{\mu}+g_{\mu 5} \mathrm{~d} x^{\mu} \mathrm{d} x^{5}+g_{55} \mathrm{~d} x^{5} \mathrm{~d} x^{5} .
$$

But it is equally natural to ask for such an equation attached to 'planes'. Because of the fact that point and plane coordinates are mutually dual one would probably recover 
similar equations. For an projective approach to duality and Classical Mechanics see 9. 10]. A methodological approach to a projective formulation of physics from nonmetrical grounds is given in [26, 28]. The most interesting point, however, is to ask for a self dual description. In projective 3 -space (this will be important later on) this leads naturally to 'lines', since lines are dual to lines. It is possible to develop projective theory from lines. Even projective differential geometry should be developed before the advent of the metrical theories. Indeed, one can find an emphatical such statement in the book of E. Wilczynski [62], dated 1906, so protected from an infection by the Einstein-Hilbert metrical theory, a virus? Moreover, one should not forget, that the natural space to investigate differential equations is projective space, as developed by Lie and Engel [44]. Point and contact transformations have there their origin.

The second virus was already discussed above, and could be called the manifold Virus:

PV/M: 'Physics takes place on a Riemannian Manifold'.

This was the belief of Kaluza, see the citation above. We have obviously non-Riemannian manifolds currently employed in physics. Fiber-bundles with non-Abelian gauge groups, super-spaces, non-commutative geometries, quantum field theories using strings, branes or M-theory, but all of them are 'point' theories developed in this spirit, especially local quantum field theory, but see below.

\subsection{Projective Quantum Theory}

We can give only cursory arguments, some more (and orthogonal) arguments can be found in $[28]$.

In e.g. [6, [7] and his book [5] Cartan developed the theory of projective spaces and spinors. It is worth to note, that Cartan used spinors for algebraic and geometric reasons in a time when they had not even be known in physics. He showed furthermore, that one needs spinors to find all irreducible representations of the classical groups. So, spinors are the most natural and useful tool to study null-invariance and quadrics in projective spaces. Null-invariance is a concept of the space of linear complexes in line geometry [56, 57.

Already in [1] Birkhoff and von Neumann showed that quantum mechanics is based on projective concepts. One has to note that the 'scalar product' of quantum mechanics is in fact a dual product, or pairing between space and dual space, which contains no metrical information:

$$
<\mid>: \mathcal{H}^{*} \times \mathcal{H} \longrightarrow \mathbb{C},
$$

where $\mathcal{H}^{*}$ is the dual space. One should note that the late Dirac told, that projective reasonings led him to find his electron equation in 1928 [52]. Dirac was furthermore interested in a projective formulation of Hamilton's principle [12] and the usage of homogenous variables in classical physics. Indeed, it is possible to find a dual and projective formulation of classical mechanics and symplectic geometries [11. Projective considerations seems to have played a major role in electron physics, a fact which is not well recognized because Hilbert space methods cannot clearly point this out

Quantum physics is incidence physics : If one analyses the mathematics of the quantum mechanical apparatus [60, it is clear that in principle a system can only be prepared at a time and measured at a second time. No information at a third time is available in principle. The time evolution has to be a free undisturbed evolution of the given dynamics described by a suitable Hamiltonian. If we denote the events of 
preparation and measurements as 'points', their unknown connection can be addressed as a 'line'. Such a line might in no way be straight. Paths in Bohmian mechanics or streamlines of the Dirac fluid show quite complicated patters. However, the important fact is that by construction the Hamiltonian has to be a linear operator and generates the time development. Moreover, to fix a 'higher order' (i.e non-geodesic) curve would necessarily involve at least a third 'point' to be known. In this sense a virtual connection between preparation and measurement can be addressed as a 'line'. Looking at the geodesic on a sphere, which are great circles, one observes that they are linearly parametrized by the angle. Only if one projects these 'lines' by a non-linear, say stereographic, projection on a plane 'lines' become 'curves' in this plane w.r.t. the distance measure there. The mere notation

$$
\psi(x, t):=e^{i \hat{\mathcal{H}} t} \psi(x, 0)
$$

is essentially of the same type.

\subsection{Algebraization of QFT}

A little bit surprising might be the fact that the method of 'second quantization' exhibits a projective structure. While the fermionic creation operators $a_{i}^{\dagger}$ span a linear space $V$ their duals, the annihilation operators, are elements of the dual space $V^{*}$. The Fock 'inner product' is thus once more a dual product

$$
\begin{aligned}
<\mid>: & \bigwedge\left(V^{*}\right) \times \bigwedge(V) \longrightarrow \mathbb{C} \\
& <n|m>=<0| a_{i_{1}} \ldots a_{i_{n}} a_{j_{1}}^{\dagger} \ldots a_{j_{m}}^{\dagger} \mid 0>=\delta_{n, m}
\end{aligned}
$$

in an obvious notation. The advantage of such a point of view is, that it allows an easy formulation of QFT in terms of the associated Clifford algebras (symplectic Clifford algebras or Weyl algebras for bosons) [15, 20]. Such a formulation is equivalent to 'functional quantum field theory' as developed and successfully utilized in [58]. Resisting $\mathrm{MV} / \mathrm{Q}$, we note that Clifford algebras of dual-products do not depend on a metrical structure.

Much more surprising might be that a thoughtful formulation of QFT in Clifford terms avoids a common singularity of QFTs due to the transition from one ordering to another, see 21, 20, 23. It seems to be a general rule that those concepts of QFT which are geometrizable are 'sane' while non-geometrizable operations, as e.g. the Bethe-Salpeter equation, are 'insane'. But, the large amount of geometrical theorems leads to unsolved questions in QFT when transfered in this direction.

\subsection{Geometric Algebras}

A main problem in dealing with geometrical properties is the mathematical axiomatization of these notions. Indeed, projective geometry and algebra are brother and sister originating from the same problems. For our purpose, we mention Graßmann 25] and Clifford [8], while Möbius, Plücker, Poncelet, Lindemann, Cayley and Klein, to cite only few, played also an important part. The main point for our argumentation is, that and algebraic description was developed which allowed to manipulate symbolically and without the Cartesian coordinates algebraic objects. The birth of algebraic geometry. 
Clifford Geometric Algebras : Unfortunately projective geometry became oldfashioned and doomed away. Only a few persons, among them D. Hestenes see e.g. [33, 35, 36], had a continuous interest in 'Geometric Algebra'. This was the term Clifford used for Clifford algebras. However, there is not a single geometric algebra, but algebraic structures are dependent on the semantic context and can be used to model geometric situations only after algebraic terms have been tied to geometric meanings 22 .

Nevertheless, we find lots of metrical applications of Clifford geometrical algebra e.g. in Lie group theory [14. But Graßmann developed his system in a projective background, as Clifford did also.

Important improvements have been achieved in a series of papers which develop a Clifford geometric formulation of quantum field theories [21, 15, 20]. The main tool in such a framework is the so-called quantum Clifford algebra, already studied in 48, 49, 19. 1] and properly defined in [22]. However, all these treatments of quantum Clifford algebras share the methodological problem that the algebras are constructed starting with vector variables and seem to imply a metrical interpretation. This can be noticed in the the frequent statements -preconception or virus- that: "Clifford algebras are metrical algebras", a variant of MV/Q!

Graßmann-Cayley and Hopf Algebras : A quite different approach starts with incidence relations which can be coded in the lattice of subspaces of a linear space, see [3, 13, 37]. One defines algebraically two operations called 'join' and 'meet' describing the linear span of two subspaces, two points span a line (join) etc. or their intersection, two lines may or may not intersect (meet) in a point. Putting some rules how to calculate, one obtains a Graßman-Cayley (GC) algebra 3. This algebra has also non geometrical applications in the theory of determinants, combinatorics etc., or the other way around, this theory provides a geometrization of large parts of mathematics. It is of recent interest, that the meet in GC algebras can be calculated in an straight forward manner using Hopf algebra techniques. The meet (join) can be seen to be related to a co-product on the dual space.

It was Gian-Carlo Rota, who developed the theory of Peano space [3] and GC algebra utilizing Hopf algebras. The co-product of a Hopf algebra is needed to compute in a clean way the meet, which cannot be done using Graßmann algebra alone. This is a long ongoing and still present story starting with Graßmann's regressive product, which lowers the grade. The most remarkable fact is, that by Cliffordization [53] one can turn a GC algebra into a Clifford algebra. This process is intimately related to the normal-ordering process of quantum field theory [23. Moreover, using the Hopf algebra antipode, Connes and Kreimer [42] have been able to produce the counter terms of perturbative renormalization. For short, Feynman diagrams may be understood as tangles of certain Clifford or Graßmann Hopf algebras: annihilation is the 'product', creation is the 'co-product' and physics is done by convolution.

All this is a complicated not yet fully understood relation, but it emerges directly from projective geometry which is recovered in the structure of quantum fields. Some of this observations belong already to the next part.

\section{3 - and Outlook}

Jordan isomorphism theorem versus Inönü-Wigner group contractions : One of the basic problems of a 5 or higher dimensional Kaluza-Klein theory is the way back to 
the observed $3+1$ dimensions. The main point of such a development is an mechanism which breaks a symmetry into a semi-direct product of sub-symmetries. A common example is the non-relativistic limit $c \rightarrow \infty$ of the Lorentz group, a classical group, into the not semi-simple Galileo group. Jordan, in reducing the 5 dimensional Kaluza-Klein theory splited off the scale transformations which have no effect on homogeneous coordinates, see 45. Since a projective point is defined in a metrical model by an $n+1$ tuple of coordinates $\mathbb{P}^{n} \ni x=\left(x_{1}, \ldots, x_{n+1}\right)$ modulo a constant $\rho$ and not all $x_{i}=0$. One finds $\rho\left(x_{1}, \ldots, x_{n+1}\right)=\left(x_{1}, \ldots, x_{n+1}\right) \bmod \rho$ for such points. It is therefore necessary to extract the transformations of the $\left(x_{1}, \ldots, x_{n+1}\right)$ which do not respect this properties. The relevant groups are $G L(n+1)$ reducing to $P G L(n)$.

It might be possible, and works for certain cases, that the Jordan isomorphism theorem should be replaced by Wigner-Inönü or Saletan contraction of symmetry groups. For a discussion see [54, 24. The direction is to proceed from a higher symmetry group to subgroups by performing a certain limit. Well known is the case:

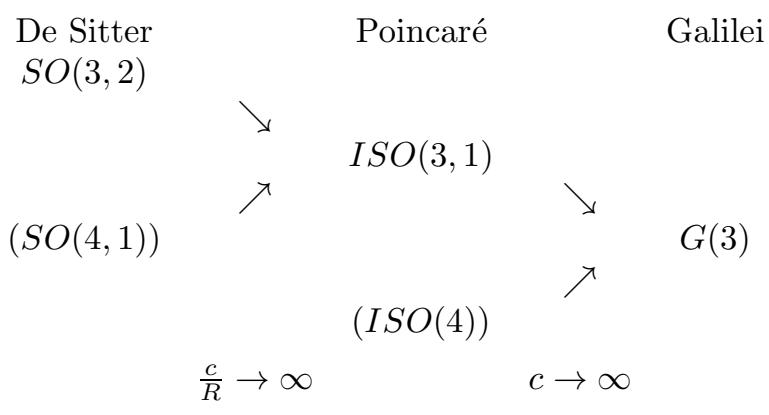

The direction in this type of contraction is from the simple group (also providing simple equations and physical laws) to more complicated structures. While one has no translations in the De Sitter group, such transformations occur in the inhomogeneous Poincaré group. Breaking down once more the group, yields the Galilei group. But the dynamical equations become tremendous complicated due to 'relativistic corrections' and even wrong if the limit $(c \gg v)$ is not valid.

Furthermore such contractions occur also in quantum physics. One can obtain the Heisenberg algebra from the following contraction scheme, where $J_{+}, J_{-}, J_{3}, \xi=I$, generating $S L(n)$, is contracted:

$$
\left[\begin{array}{c}
h_{+} \\
h_{-} \\
h_{3} \\
I
\end{array}\right]=\left[\begin{array}{cccc}
\alpha & & & \\
& \alpha & & \\
& & 1+\frac{\beta}{2 \alpha} & \\
& & & 1
\end{array}\right] \quad\left[\begin{array}{c}
J_{+} \\
J_{-} \\
J_{3} \\
\xi
\end{array}\right]
$$

Identifying $h_{3}=N, h_{+}=a^{\dagger}, h_{-}=a$ and $I=I$, we obtain the usual oscillator algebra $\left[N, a^{\dagger}\right]_{-}=a^{\dagger},[N, a]_{-}=-a$ and $\left[a^{\dagger}, a\right]_{-}=I$. The same process takes place in the 'contraction' of the 5 -dimensional projective Kaluza-Klein theory. In algebraic terms, this is a process going from finer to coarser gradings in the algebras by factoring out certain even sub-algebras. Hestenes [35] after Weyl [61] calls this a space-time split. A projective interpretation of the Lorentz group is also possible [26, 11].

Metrics from 6-vectors A recent program tries to involve so called 6-vectors to derive metric fields [29, 30, 31, 47]. Starting point is electrodynamics in media. The linear relation assumed between $\vec{E}, \vec{D}$ and $\vec{H}, \vec{B}$ is then sufficient to induce a Minkowski metric. However, a 6 -vector $(\vec{E}, \vec{B})$ is a screw [2], and screws played an important role 
in the mechanics of rigid bodies in curved space [63]. Following up this idea, on comes to two loose ends. Screws are spinors and screws are special linear complexes. The approach of [31] employs thus line geometry, and with Felix Klein one says that 'line geometry is metrical geometry' (on the Null quadric). The next paragraph will try to connect this again to projective geometry.

Null-invariance and Field equations A radical projective approach has to provide own arguments to find field equations which should not depend on metrical properties. Till now we rejected the metric theory of Einstein-Hilbert in 5-dimensional space, but we gave no alternative. But it is known, that $\stackrel{5}{R}_{\mu \nu}=0$ is needed to obtain the (almost) correct contraction to usual 4-dimensional Einstein-Hilbert-Maxwell theory.

In the last paragraph we noted, that 6 -vectors are special linear complexes, see also 27, 62]. In addition, our examples of projective geometry have been of 3-dimensional projective space which is described by 4 homogenous coordinates. This seems to be not related to 5-dimensional Kaluza-Klein theories. However, the space of lines is already four dimensional, and the space of linear complexes constitutes a 5-dimensional projective space which can be described by 6 -vectors. If one demands, that the incidence relations of the 5-dimensional projective space of linear complexes can be reduced to the space of 3-dimensional projective space including the incidence relations, one obtains a null-invariance [56, 57]. The null-invariance describes a quadric and a metrical surface. If the infinite elements are moved, the quadric becomes dynamical and the metric itself a field. The null-invariance is exactly given by an equation like $\stackrel{5}{R}_{\mu \nu}=0$, which we propose to re-interpret in this way. However, a deep understanding of this process will need a much better understanding of the different duality structures in GC algebras including their Hopf algebra nature, which is intimately linked to the incidence structure.

\section{Conclusion}

We gave a selection of arguments, others are available, which are quoted to point out different aspects of projective reasoning and its connection to different fields of physics. Adding them together yields a picture from a puzzle if correctly assembled.

We proposed to replace the Jordan isomorphy theorem by Wigner-Inönü or Saletan contractions of Lie groups. Furthermore one should interpret the 'vacuum' field equations of 5-dimensional Kaluza-Klein theory as the statement of null invariance in the 5 -dimensional projective space of linear complexes. This is a radical change in the usually adopted interpretation of Kaluza-Klein theories. We start from projective 3-space, no artificial higher dimensional 'world dimensions' are introduced. But, this restricts our approach to 5-dimensional Kaluza-Klein theory. Such a reasoning is in accord with the approach of [31, 47].

This program is not only motivated by projective relativity theory, but also by the puzzling fact that quantum field theory has via its recently observed Hopf algebraic structure a close relation to projective geometry too! Perhaps, one cannot unify relativity and quantum mechanics, but there is already a glimpse of a projective framework capable to describe both structure. This article is a first small step in this new direction.

\section{Acknowledgement}

I would like to thank Prof. G. Lessner for fruitful discussions. 


\section{References}

[1] R. Abłamowicz, P. Lounesto; On Clifford algebras of a bilinear form with antisymmetric part, in "Clifford algebras with numeric and symbolic computations", R. Abłamowicz, P. Lounesto, J.M.Parra Eds., Birkhäuser, Boston, 1996, 167-188

[2] R.S. Ball; Some extensions on the theory of screws, Trans. Roy. Irish Acad. 32, 1904, 299-366

[3] M. Barnabei, A. Brini, G.-C. Rota; On the exterior calculus of invariant theory, J. Alg. 96 1985, 120-160

[4] G. Birkhoff, J. von Neumann; Ann. of Math. 37(4) (1936) 823

[5] É. Cartan; The theory of spinors, Hermann, Paris 1966 reprint by Dover, New. York 1981

[6] É. Cartan; Les Groupes projective qui ne laissent invariante aucune Multiplicité plane, Bull. de la Soc. Math. de France T.41, 1913 53-96

[7] É. Cartan; Le Principle de Dualité et la Théorie des Groups simple et semi-simple, Bull. des Sci. Math. Sér. 2 (49) 1925 361-374

[8] W.K. Clifford; Mathematical papers, reprinted from the 1882 edition by Chelsea Publ. Comp., New York 1968

[9] O. Conradt; The Principle of duality in Clifford algebra and projective geometry, in "Clifford algebras and their applications in mathematical physics", R. Abłamowicz, B. Fauser Eds. Birkhäuser, Boston 2000, 157-193

[10] O. Conradt; Mechanics in Space and Counterspace, J. Math. Phys. 41(10) 2000 6995-7028

[11] O. Conradt; Mathematical Physics in Space and Counterspace, Thesis, University of Basel, Aug. 2000

[12] P.A.M. Dirca; Homogenous variables in classical dynamics, Proc. Camb. Phil. Soc. 291933 389-400

[13] P. Doubilet, G.-C. Rota, J.A. Stein; On the foundation of combinatorial theory: IX Combinatorial methods in invariant theory, Studies in Appl. Mathematics, Vol LIII(3), 1974, 185-216

[14] C. Doran, D. Hestenes, F. Sommen, N. Van Acker; Lie groups as spin groups, J. Math. Phys. 34(8), 1993, 3642-3669

[15] B. Fauser; Clifford-algebraische Formulierung und Regularität der Quantenfeldtheorie, Thesis, Uni. Tübingen, 1996.

[16] B. Fauser, H. Stumpf; Positronium as an Example of Algebraic Composite Calculations, in Proc. of "The Theory of the Electron", J. Keller, Z. Oziewicz Eds., Cuautitlan, Mexico, 1995, Adv. Appl. Clifford Alg. 7 (Suppl.), 1997, 399-418.

[17] B. Fauser; Clifford geometric parameterization of inequivalent vacua, Submitted (hep-th/9710047). 
[18] B. Fauser; Dirac theory from a field theoretic point of view, Proc. "Clifford algebras and their applications in mathematical physics", Aachen 1996, V. Dietrich, K. Habetha, G. Jank Eds., Kluwer, Dordrecht 1998

[19] B. Fauser; Clifford algebraic remark on the Mandelbrot set of two-component number systems, Adv. Appl. Clifford Alg. 6 (1), 1996, 1-26.

[20] B. Fauser; On an easy transition from operator dynamics to generating functionals by Clifford algebras, J. Math. Phys. 39, 1998, 4928-4947.

[21] B. Fauser; Vertex normal ordering as a consequence of nonsymmetric bilinear forms in Clifford algebras, J. Math. Phys. 37, 1996, 72-83.

[22] B. Fauser, R. Ablamowicz; On the decomposition of Clifford algebras with arbitrary bilinear form in "Clifford algebras and their applications in mathematical physics", Vol. 1, Algebra and general, R. Abłamowicz, B. Fauser eds. Birkhäuser, Boston 2000

[23] B. Fauser; On the Hopf algebraic origin of Wick normal-ordering, hep-th/0007032, submitted

[24] R. Gilmore; Lie groups, Lie algebras and some of their applications, WileyInterscience, New York, 1974

[25] H. Grassmann; Die lineale Ausdehnungslehre [1844], 2nd. ed. with notes by Grassmann, Verlag Otto Wigand, Leipzig, 1878

[26] P. Gschwind; Raum Zeit Geschwindigkeit, Mathematisch-Astronomische Sektion am Goetheanum, Dornach CH, 1986

[27] P. Gschwind; Der lineare Komplex - eine überimaginäre Zahl, PhilosophischAnthroposophischer Verlag am Goetheanum, Dornach CH, 1991

[28] P. Gschwind; Methodische Grundlagen zu einer projektiven Quantenmechanik, Philosophisch-Anthroposophischer Verlag am Goetheanum, Dornach CH, 1989

[29] G. Harnett; Metrics and dual operators, J. Math. Phys. 31(1) 1991 84-91

[30] G. Harnett; The bivector Clifford algebra and the geometry of Hodge dual operators, J. Phys. A: MAth. Gen. 251992 5649-5662

[31] F.W. Hehl, Y.N. Obukhov, G.F. Rubilar; Spacetime metric from linear electrodynamics II, preprint gr-qc/9911096

[32] G. Hessenberg; Vektorielle Begründung der Differentialgeometrie, Math. Ann. Bd. 78, 1917, 187-217

[33] D. Hestenes; Space Time Algebra, Gordon and Beach, 1966

[34] D. Hestenes; Mathematical Viruses, in "Clifford algebras and their applications in mathematical physics" Montpellier 1989, A. Micali, R. Boudet, J. Helmstetter, Eds., Kluwer Acad. Publischers, Dordrecht, 1992, 3-16

[35] D. Hestenes; The design of linear algebra and geometry, Acta Appl. Math. 23, 1991, 65-93 
[36] D. Hestenes, R. Ziegler; Projective geometry with Clifford algebra, Acta Appl. Math. $23,1991,25-64$

[37] S.A. Joni, G.-C. Rota; Coalgebras and bialgebras in combinatorics, Studies in Appl. Math. 61, 1979, 93-139

[38] P. Jordan; Schwerkraft und Weltall, Vieweg und Sohn, Braunschweig 1955

[39] L. Kadison, M.T. Kromann; Projective Geometry and Modern Algebra Birkhäuser / Boston 1996

[40] Th. Kaluza; Zum Unitätsproblem der Physik, Sitzungsber. d. Berl. Akad. 1918204

[41] Th. Kaluza; On the unity problem of physics, in "Modern Kaluza Klein theories" Th. Appelquist, A. Chodos, P.G.O. Freund Eds. Addison-Wesley Publ. Comp. Inc. 1987, p. 61

[42] D. Kreimer; Knots and Feynman diangrams, Cambridge Lecture Notes in Physics, Cambridge Univ. Press, Cambridge 2000

[43] G. Lessner; Unified field theory an the basis of the projective theory of relativity, Phys. Rev. D 25(12), 1982, 3202-3217

[44] S. Lie, F. Engel; Theorie der Transformationsgruppen, Teubner, Leipzig, 18881893, Vol.I,II,III

[45] G. Ludwig; Fortschritte der projektiven Relativitätstheorie, Fr. Vieweg und Sohn, Braunschweig, 1951

[46] A. Macias, H. Dehnen; Dirac field in the eight-dimensional Kaluza-Klein theory, Mod. Phys. Lett. A Vol 7, No. 2 1992, 103-116

[47] Y.N. Obukhov, F.W. Hehl; Spacetime metric from linear electrodynamics I, preprint gr-qc/9904067

[48] Z. Oziewicz; From Grassmann to Clifford in Proceedings "Clifford Algebras and their Application in Mathematical Physics", Canterbury, UK, J.S.R. Chisholm, A.K. Common, Eds., Kluwer, Dordrecht, 1986, 245-256.

[49] Z. Oziewicz; Clifford algebra of multivectors, Proc. Int. Conf. on "The Theory of the Electron", (Cuautitlan, Mexico, 1995). J. Keller and Z. Oziewicz Eds., Adv. in Appl. Clifford Alg. 7 (Suppl.), 1997, 467-486.

[50] W. Pauli; Über die Formulierung der Naturgesetzte mit fünf homogenen Koordinaten, Teil I: Klassische Physik, Ann. Phys. Bd. 18, 1933, 305-336

[51] W. Pauli; Über die Formulierung der Naturgesetzte mit fünf homogenen Koordinaten, Teil II: Die Diracschen Gleichungen f"ur Materiewellen, Ann. Phys. Bd. 18, 1933, 337-372

[52] H. Rechenberg; The foundations of geometry and quantum algebra - From Dirac's early works on quantum mechanics, Special Volume dedicated to the life and scientific activities of P.A.M. Dirac, Academy of Sciences of the USSR, Moscow (Preprint of the MPI Munich Germany, MPI-PAE/PTh 32/87 April 1987)

[53] G.-C. Rota, J.A. Stein; Plethystic Hopf algebras, Proc. Natl. Accad. Sci. USA, Vol 91, 1994, 13057-13061 
[54] H. Saller, D. Finkelstein; From complicated to simple or from Heisenberg to Clifford or from contractions to normalizations, unpublished manuscript 1999

[55] E. Schmutzer; Relativistische Physik, Teubner Verlagsgesellschaft, Leipzig 1968

[56] H.-J. Stoß; Treffgeraden und Nullinvarianz, Beiträge zur Lienengeometrie, Philosophischer -Anthroposophischer Verlag am Goetheanum, Dornach CH, 1995

[57] H.-J. Stoß; Einführung in die synthetische Liniengeometrie, Philosophischer Anthroposophischer Verlag am Goetheanum, Dornach CH, 1999

[58] H. Stumpf, Th. Borne; Composite particle dynamics in quantum field theory, Vieweg und Sohn / Braunschweig 1994

[59] J.G. Vargas, D.G. Torr; Clifford-valued Clifforms: A geometric language for Dirac equations, in "Clifford algberas and their applications in mathematical physics" R. Abłamowicz, B. Fauser Eds., Birkhäuser, Boston 2000, 135-154

[60] J. Verhulst; Der glanz von Kopenhagen, Geistige Perspektiven der modernen Physik, Verlag Freies Geistesleben, Stuttgart 1994

[61] H. Weyl; Raum Zeit Materie, hrsg. u. erg. von Jrgen Ehlers Berlin, Springer, Heidelberg, 1988

[62] E.J. Wilczynski; Projective differential geometry of curves and ruled surfaces, Teubner, Leipzig 1906

[63] R. Ziegler; Die Geschichte der Klassischen Mechanik im 19. Jahrhundert, Franz Steiner Verlag Wiesbaden, Stuttgart 1985 